\title{
Radiation-Induced Malignant Peripheral Nerve Sheath Tumor of the Vagus Nerve Following Radiation Treatment of Cervical Paraganglioma
}

\author{
Gregory P. Lekovic ${ }^{1}$ Gautam U. Mehta1® Anne K. Maxwell ${ }^{2}$ Kevin A. Peng ${ }^{2}$ Derald E. Brackmann ${ }^{2}$ \\ ${ }^{1}$ Division of Neurosurgery, House Institute, Los Angeles, California, \\ Address for correspondence Gregory P. Lekovic, MD, PhD, House \\ United States \\ 2 Division of Neurotology, House Institute, Los Angeles, California, \\ Clinic, 2100 West Third Street, Los Angeles, CA 90057, United States \\ United States
}

J Neurol Surg Rep 2020;81:e66-e70.
Abstract
Keywords
- paraganglioma
- radiation
- vagus nerve
- malignant peripheral nerve sheath tumor
- radiation-induced sarcoma
- glomus vagale

Radiation-induced sarcoma is a known but rare complication of radiation treatment for skull base paraganglioma. We present the cases of a female patient with multiple paraganglioma syndrome treated with external beam radiation treatment who presented 4 years later with a malignant peripheral nerve sheath tumor of the vagus nerve.

\section{Case Report}

A 28-year-old woman with a known history of multiple paraganglioma syndrome presented with enlarging mass in the right neck, dysphonia, cough, dysphagia, and severe neck pain. Weight loss was attributed to poor oral intake secondary to dysphagia and nausea. Four years previously, the patient was diagnosed with bilateral carotid body tumors. She completed a course of 6 weeks of external beam radiation therapy (EBRT) at that time for the rightsided tumor which was felt to be inoperable due to rostral extension to the skull base. The prescription dose was 5,400 cGy delivered in 180 cGy fractions. She subsequently had a left-sided carotid body tumor removed approximately 1 year later with no adverse sequelae. Both procedures were performed at an outside hospital. After radiation treatment, the patient had a partial imaging response and reported improvement in local symptoms until 4 years later, when she noticed progressive dysphonia, dysphagia, and pain. The patient was not evaluated for succinate dehydrogenase (SDH) mutation or other germline genetic analysis.

On physical examination, a firm mass was palpable in the upper neck. The patient had deviation of the soft palate/uvula to the left consistent with right-sided glossopharyngeal nerve deficit. On direct laryngoscopy, she was found to have an immobile right vocal cord consistent with vagus nerve palsy. The remainder of the patient's neurologic examination was unremarkable. In addition, the patient did not have café-au-lait macules, freckling of the axilla or groin, cutaneous or subcutaneous tumors, or other clinical stigmata of neurofibromatosis. received

March 16, 2020 accepted after revision September 2, 2020
DOI https://doi.org/ $10.1055 / \mathrm{s}-0040-1718408$ ISSN 2193-6358.

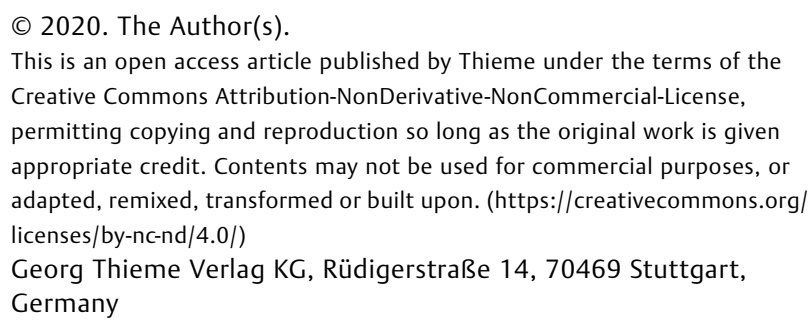




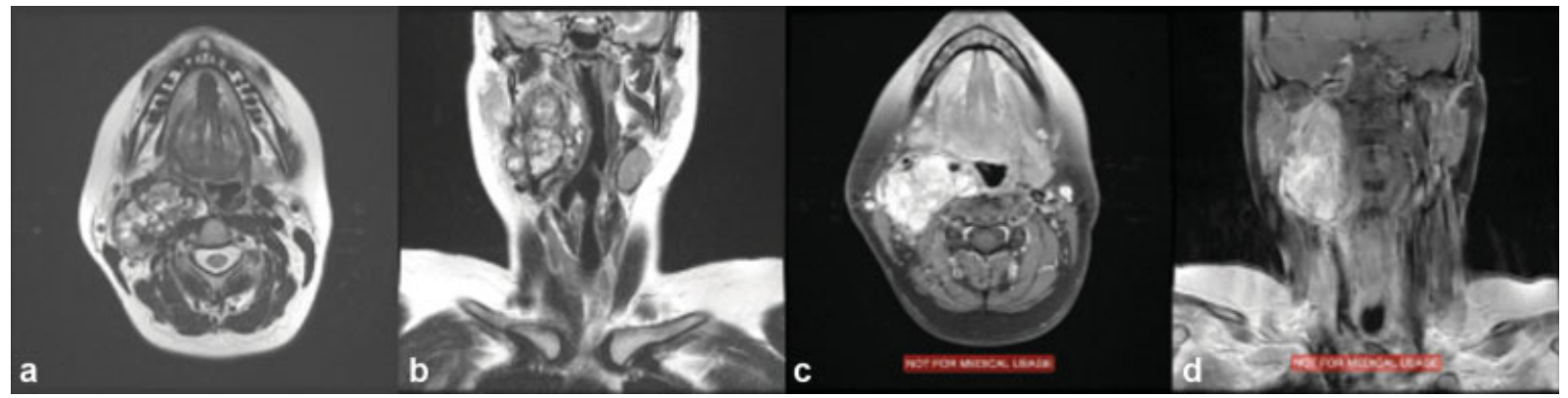

Fig. 1 Preoperative T2-weighted axial (a) and coronal (b) magnetic resonance imaging of the brain and neck showing a large mass in the carotid sheath with "popcorn"-type appearance of flow voids suggestive of paraganglioma; T1-weighted axial and coronal images following administration of gadolinium demonstrate avid contrast enhancement and splaying of the internal and external carotid arteries (c and $\mathbf{d}$, respectively).

Magnetic resonance imaging (MRI) of the neck demonstrated a homogeneously enhancing mass in the posterior parapharyngeal space of the neck on the right side with anterior displacement of the carotid artery and internal jugular vein, extending from the carotid bifurcation inferiorly to the jugular foramen rostrally (-Fig. 1). Based on this imaging and the patient's clinical history of multiple paraganglioma syndrome, the presumptive diagnosis was that of a glomus vagale, carotid body tumor, or possibly a "collision tumor" of both. Given that the patient was symptomatic with enlarging neck mass, new neurologic deficits (i.e., glossopharyngeal and vagus neuropathies), and prior failed radiation treatment, surgery was recommended.

\section{Surgery Details}

Preoperative angiography with embolization was performed 1 week prior to surgery. The angiogram demonstrated a vascular blush typical of paraganglioma, but limited to only part of the tumor mass that enhanced on MRI (-Fig. 2). This was attributed to a partial response to radiation. A balloon test occlusion was performed, which the patient tolerated without evidence of ischemia. A gastrostomy tube was placed prior to surgery to assist with nutrition both before surgery and in anticipation of persistent postoperative dysphagia. The patient underwent a transjugular approach to the infratemporal fossa and cervical carotid artery, including blind sac closure of the external auditory canal, anterior transposition of the facial nerve, ligation of the internal jugular vein and sigmoid sinus, exenteration of the jugular foramen contents, and carotid artery sacrifice. A gross total resection of tumor was felt to have been obtained; however, surgical margins were not evaluated at the time of surgery. Four regional lymph nodes were excised for pathological evaluation of possible metastases.

\section{Pathology}

Pathology revealed two separate and distinct histologic components, including one comprised weakly eosinophilic

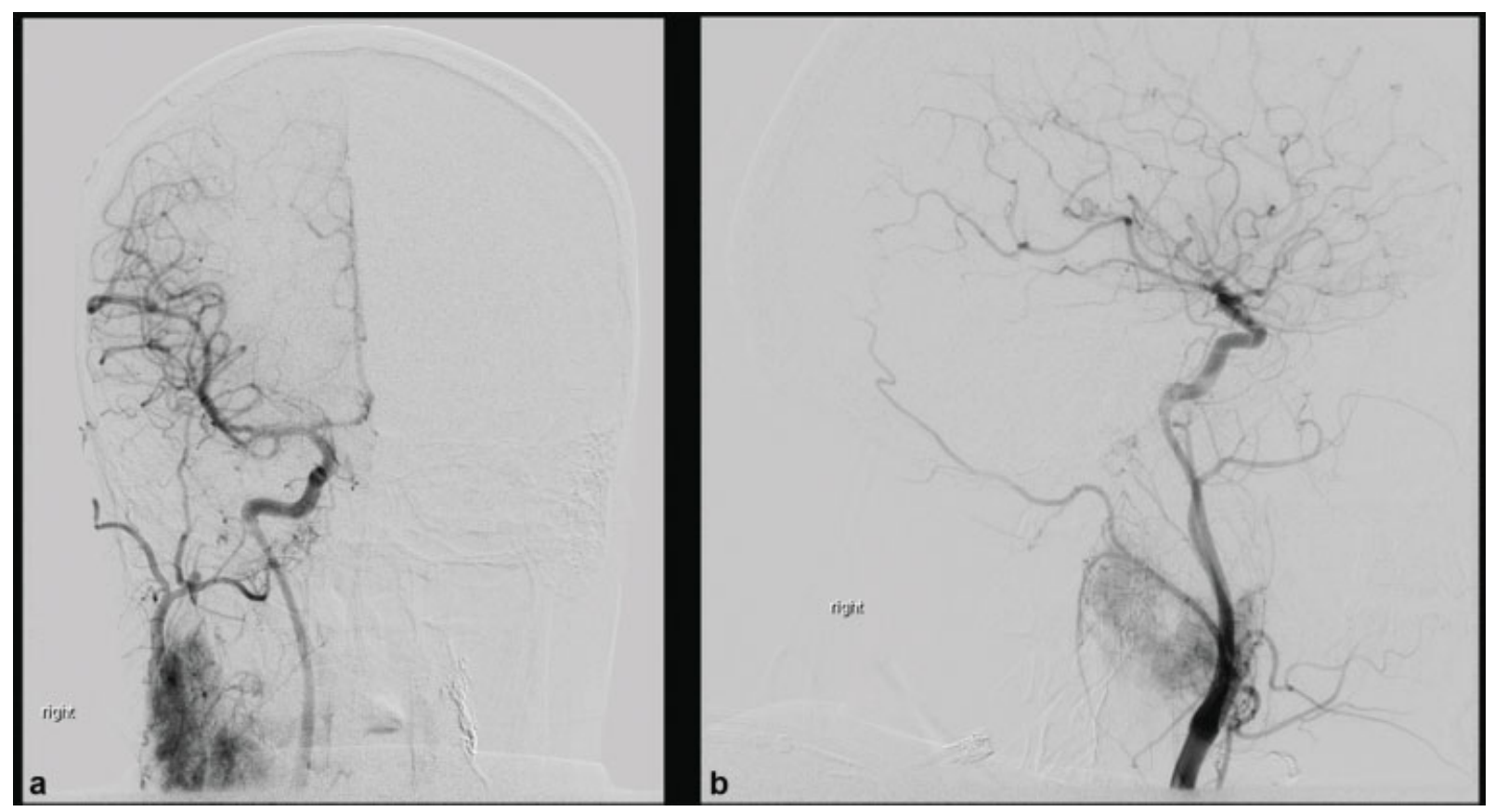

Fig. 2 Preoperative anteroposterior (a) and lateral (b) angiograms show two distinct patterns of vascularity: a prominent blush typical of paraganglioma and an avascular component with pronounced mass effect on the distal carotid artery. 

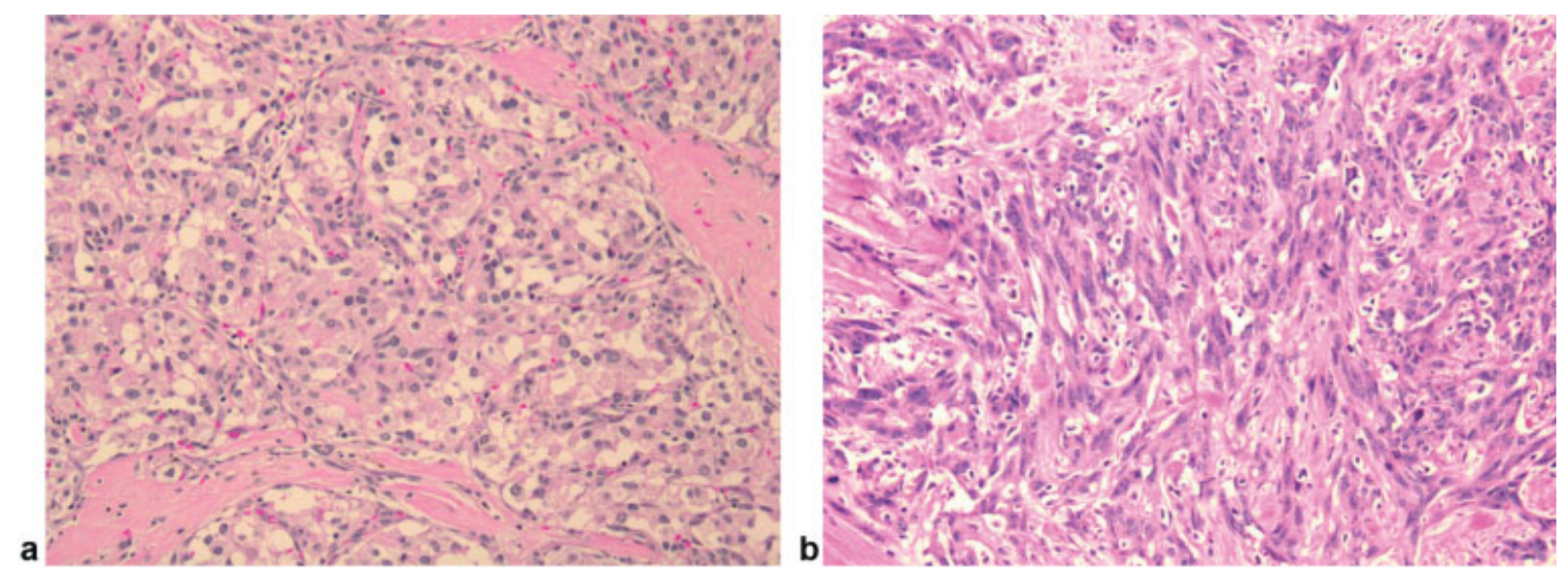

Fig. 3 Hematoxylin and eosin staining shows two distinct tumors: paraganglioma (a) characterized by "zellballen" and a spindle cell neoplasm (b). This latter tumor had a high mitotic rate and demonstrated a high $\mathrm{K}_{\mathrm{i}}-67$ staining rate (70\%) (not shown).

large cuboidal and polygonal cells arranged in clusters, or "zellballen" with supporting sustentacular cells, and an infiltrating spindle cell lesion composed of large ovoid spindle-shaped cells with prominent nucleoli and amphophilic cytoplasm (-Fig. 3). No necrosis was seen. Additional immunohistochemistry was performed that demonstrated S100 positivity in the high-grade, spindle-cell component of the tumor and chromogranin A, and synaptophysin staining in the sustentacular cells of the paraganglioma component. $\mathrm{K}_{\mathrm{i}}-67$ staining was present in $2 \%$ of the paraganglioma and $70 \%$ of the spindle cell lesion. The overall pathology impression was that of two separate contiguous tumors: a paraganglioma and also a spindle cell neoplasm consistent with high-grade malignant peripheral nerve sheath tumor (MPNST). There was no evidence of underlying plexiform neurofibroma on histopathology. Regional lymph nodes were negative for tumor.

\section{Postoperative Course}

Postoperatively, the patient developed new facial nerve weakness (House-Brackmann grade 6/6, which improved to grade 4/6 at last follow-up) but was otherwise neurologically intact. Her pain was improved, but she complained of persistent nausea and vomiting. She was discharged home and referred to speech and language pathology. Given the diagnosis of MPNST, staging computed tomography (CT) was obtained and multiple pulmonary nodules were identified. A CT-guided biopsy confirmed metastatic MPNST to the lung. The patient was referred to oncology and completed an initial regimen of vincristine, doxorubicin, and cyclophosphamide. Additional metastatic lesions were subsequently identified in the left ilium and at L2. A second round of chemotherapy with doxorubicin and ifosfamide was completed with partial response of systemic metastatic disease but with progression of locally recurrent disease in the jugular foramen and cerebellopontine angle. The patient had multiple chemotherapy-related complications, including neutropenia with opportunistic fungal infection and fungemia, and thrombocytopenia. Given the rapid local progression of disease and poor performance status, the patient was transitioned to palliative care. She expired 2 months thereafter ( 6 months after surgery).

\section{Discussion}

Radiation therapy is an established treatment option for patients with skull base paragangliomas. ${ }^{1,2}$ Tumor control (defined as cessation of tumor growth, including both stable and shrinking tumors) is achieved in 88 to $100 \%$ of patients following radiation therapy, with fewer cranial nerve complications compared with surgery. ${ }^{3-5}$ Radiation treatment options for patients undergoing radiation therapy include both conventional EBRT and stereotactic radiosurgery (SRS), sometimes called stereotactic body radiation therapy (SBRT). EBRT for paraganglioma has decades of proven efficacy at achieving tumor control. ${ }^{6-9}$ Although most complications associated with conventional radiation therapy are not life threatening, induction of malignancy following EBRT for paraganglioma may also occur. ${ }^{1,2,10,11}$

\section{Radiation-Induced Malignant Peripheral Nerve Sheath Tumor}

The association between radiation therapy and subsequent development of sarcoma has been appreciated since Cahan et al's original report in 1948 of 11 sarcomas of bone following irradiation. ${ }^{12}$ Approximately $6 \%$ of all head and neck sarcomas occur in patients with a history of prior irradiation, ${ }^{13}$ with leiomyosarcoma and other subtypes of sarcoma more commonly seen with a history of prior irradiation. ${ }^{14}$ Conversely, although MPNSTs are rare, roughly $10 \%$ of all MPNST occur in patients with a history of prior irradiation and are associated with high local recurrence and poorer overall prognosis. ${ }^{15}$

Our case satisfies Cahan et al's criteria for radiationinduced sarcoma: (1) the malignancy arose from the irradiated field; (2) the malignancy was of a different histology than the neoplasm treated with irradiation; (3) sufficient delay exists between the radiation exposure and development of secondary malignancy; and (4) the secondary malignancy arose from normal in-field tissue. Latency in our 
case between radiation exposure and sarcoma development is just over 4 years, consistent with Cahan et al's original criteria but less than the median reported by others. $^{13,14,16-19}$ This may be attributable to the patient's relative young age and the possible presence of a genetic tumor predisposition syndrome. The final Cahan et al's criteria, that the induced neoplasm arises from tissue "normal" prior to treatment, are in this case speculative. Importantly, we do not have the patient's initial presenting imaging (i.e., prior to radiation therapy), and hence cannot unequivocally rule out the presence of a coexisting neurofibroma of the vagus nerve. At surgery, the tumor specimen consisted of both paraganglioma and MPNST both involving the vagus nerve. It is possible that the patient previously had both a carotid body tumor and glomus vagale on that side, although this may not have been appreciated at the time of initial treatment. In any event, the vagus nerve would be in contact with the paraganglioma from the skull base to carotid bifurcation. Although biopsy was not obtained prior to radiation treatment on the patient's right side, she had a history of prior surgery for carotid body tumor on the contralateral side, confirming the diagnosis of multiple paraganglioma syndrome. Furthermore, at the time of radiation treatment, the patient displayed no signs or symptoms of MPNST, only later developing (e.g., severe pain). Nevertheless, without preoperative imaging and genetic analysis (see later), it remains theoretically possible that the patient had either coexisting neurofibromatosis type 1 (NF1) and multiple paraganglioma syndrome or had a pathological mutation contributing to a mixed phenotype. If so, the induction of a secondary malignancy may not fully fulfill Cahan et al's criteria for malignant transformation of "normal" in field tissue.

\section{Role of Radiation Therapy Technique in Induction of Malignancy}

A dose-response relationship between radiation exposure and induction of malignancy has been well established in both experimental models and clinically, such as in survivors of atomic bomb exposure. ${ }^{17,20}$ Whether there is a threshold of exposure for cancer induction, and the shape of the doseresponse curve for higher radiation exposures, however, is not well established. It has been postulated that for hematological malignancies, reduced cell survival seen with higher doses of therapeutic radiation leads to a decrease in malignancy induction with high doses, that is, that the doseresponse relationship is "bell shaped." ${ }^{17,19}$ In contrast, sarcoma induction is more commonly seen after high radiation doses, with evidence of an increased risk of secondary malignancy with doses up to $60 \mathrm{~Gy} .^{13,16,21}$ This dose dependence may be clinically relevant when comparing SRS with EBRT: In a large study of sarcoma risk following breast irradiation, Rubino et al found the risk of sarcoma 30.6 times higher for doses more than $44 \mathrm{~Gy}$ compared with $15 \mathrm{~Gy} .{ }^{22}$ SRS allows for highly conformal treatment plans resulting in minimal dose to surrounding tissues and achieves rates of tumor control comparable to conventional (Guss et al, 2011). ${ }^{3,23-27}$ Importantly, the mean prescription dose for patients treated with SRS (median marginal doses between $1,400$ and $1,730 \mathrm{cGy})^{14}$ is significantly less than with traditional EBRT regimens such as were employed with our patient (5,400 cGy in $180 \mathrm{cGy}$ fractions). As SRS achieves tumor control comparable to that obtained with EBRT with a significantly lower prescription dose, SRS might have a lower risk of induction of malignancy when treating head and neck paragangliomas. However, given the rarity of head and neck paraganglioma and the infrequency with which SRS has historically been used for these tumors, there is insufficient data in the literature to make any firm conclusions regarding the relative safety of SRS versus EBRT.

\section{Role of Mutational Analysis of Paraganglioma Syndrome}

Our patient did not have genetic testing performed prior to treatment. Multiple genes/genetic pathways have been shown to contribute to head and neck paraganglioma formation, including SDH, Von Hippel-Lindau, and RAS signaling pathway genes such as NF1. ${ }^{28}$ Some SDH mutations (i.e., SDHB) are associated with a significantly increased risk of malignant paraganglioma, ${ }^{29}$ whereas NF1 mutations may predispose to MPNST, as seen in our patient. Whether a patient's specific genetic landscape poses a predictable risk factor with regard to the underlying radiation dose-response relationship of new tumor induction is however unknown. Specifically, whether patients' susceptibility to either malignant transformation or sarcoma induction is impacted by a given specific causative mutation (e.g., SDHB, NF1, etc.) is not established. Nevertheless, we believe that characterization of the causative genetic mutation(s) could have helped inform the patient's initial decision to proceed with radiation treatment (e.g., if an underlying NF1 mutation had been identified).

\section{Summary}

Clinical suspicion for malignancy in head and neck paragangliomas previously treated with radiation is warranted. Pain is an unusual symptom with paraganglioma, and as is the case with peripheral nerve tumors should raise suspicion of malignant transformation. Radiation strategies aimed at lowering overall dose such as SRS/SBRT may be preferable to conventional EBRT for patients undergoing radiation of head and neck paraganglioma. Genetic analysis should be obtained prior to initiation of treatment in the management of head and neck paraganglioma.

\section{Ethical Approval}

All procedures performed in studies involving human participants were in accordance with the ethical standards of the institutional and/or national research committee and with the Declaration of Helsinki 1964 and its later amendments or comparable ethical standards.

\footnotetext{
Patient Consent

The patient's next of kin has given consent for submission of this case report.
} 


\section{Funding}

None.

\section{Conflict of Interest}

All authors certify that they have no affiliations with or involvement in any organization or entity with any financial interest (such as honoraria; educational grants; participation in speakers' bureaus; membership, employment, consultancies, stock ownership, or other equity interest; and expert testimony or patent-licensing arrangements) or nonfinancial interest (such as personal or professional relationships, affiliations, knowledge, or beliefs) in the subject matter or materials discussed in this case report.

\section{References}

1 Galland-Girodet S, Maire JP, De-Mones E, et al. The role of radiation therapy in the management of head and neck paragangliomas: impact of quality of life versus treatment response. Radiother Oncol 2014;111(03):463-467

2 Gilbo P, Morris CG, Amdur RJ, et al. Radiotherapy for benign head and neck paragangliomas: a 45-year experience. Cancer 2014;120 (23):3738-3743

3 Liscak R, Urgosik D, Chytka T, et al. Leksell Gamma Knife radiosurgery of the jugulotympanic glomus tumor: long-term results. J Neurosurg 2014;121(Suppl):198-202

4 Cole JM, Beiler D. Long-term results of treatment for glomus jugulare and glomus vagale tumors with radiotherapy. Laryngoscope 1994;104(12):1461-1465

5 Hansen HS, Thomsen KA. Radiotherapy in glomus tumours (paragangliomas). A 25 year-review. Acta Otolaryngol Suppl 1988; 449:151-154

6 Jackson AW, Koshiba R. Treatment of glomus jugulare tumours by radiotherapy. Proc R Soc Med 1974;67(04):267-270

7 Krych AJ, Foote RL, Brown PD, Garces YI, Link MJ. Long-term results of irradiation for paraganglioma. Int J Radiat Oncol Biol Phys 2006;65(04):1063-1066

8 Lalwani AK, Jackler RK, Gutin PH. Lethal fibrosarcoma complicating radiation therapy for benign glomus jugulare tumor. Am J Otol 1993;14(04):398-402

9 Lustig LR, Jackler RK, Lanser MJ. Radiation-induced tumors of the temporal bone. Am J Otol 1997;18(02):230-235

$10 \mathrm{Na}$ AF, Lai LT, Kaye AH. Radiation induced brainstem glioblastoma in a patient treated for glomus jugulare tumour. J Clin Neurosci 2015;22(01):219-221

11 Preissig SH, Bohmfalk GL, Reichel GW, Smith MT. Anaplastic astrocytoma following radiation for a glomus jugular tumor. Cancer 1979;43(06):2243-2247

12 Cahan WG, Woodard HQ, Higinbotham NL, Stewart FW, Coley BL. Sarcoma arising in irradiated bone; report of 11 cases. Cancer 1948;1(01):3-29
13 Mark RJ, Bailet JW, Poen J, et al. Postirradiation sarcoma of the head and neck. Cancer 1993;72(03):887-893

14 Williams L, Tmanova L, Mydlarz WK, et al. Radiation-associated sarcoma of the neck: case series and systematic review. Ann Otol Rhinol Laryngol 2018;127(10):735-740

15 Wong WW, Hirose T, Scheithauer BW, Schild SE, Gunderson LL. Malignant peripheral nerve sheath tumor: analysis of treatment outcome. Int J Radiat Oncol Biol Phys 1998;42(02):351-360

16 Berrington de Gonzalez A, Gilbert E, Curtis R, et al. Second solid cancers after radiation therapy: a systematic review of the epidemiologic studies of the radiation dose-response relationship. Int J Radiat Oncol Biol Phys 2013;86(02):224-233

17 Kumar S. Second malignant neoplasms following radiotherapy Int J Environ Res Public Health 2012;9(12):4744-4759

18 Rosko AJ, Birkeland AC, Chinn SB, et al. Survival and margin status in head and neck radiation-induced sarcomas and de novo sarcomas. Otolaryngol Head Neck Surg 2017;157(02): 252-259

19 Toda K, Shibuya H, Hayashi K, Ayukawa F. Radiation-induced cancer after radiotherapy for non-Hodgkin's lymphoma of the head and neck: a retrospective study. Radiat Oncol 2009;4:21

20 Little MP. Evidence for dose and dose rate effects in human and animal radiation studies. Ann ICRP 2018;47(3-4):97-112

21 Kuttesch JF Jr, Wexler LH, Marcus RB, et al. Second malignancies after Ewing's sarcoma: radiation dose-dependency of secondary sarcomas. J Clin Oncol 1996;14(10):2818-2825

22 Rubino C, Shamsaldin A, Lê MG, et al. Radiation dose and risk of soft tissue and bone sarcoma after breast cancer treatment. Breast Cancer Res Treat 2005;89(03):277-288

23 Genç A, Bicer A, Abacioglu U, Peker S, Pamir MN, Kilic T. Gamma Knife radiosurgery for the treatment of glomus jugulare tumors. J Neurooncol 2010;97(01):101-108

24 Guss ZD, Batra S, Limb CJ, et al. Radiosurgery of glomus jugulare tumors: a meta-analysis. Int J Radiat Oncol Biol Phys 2011;81(04): e497-e502

25 Ibrahim R, Ammori MB, Yianni J, Grainger A, Rowe J, Radatz M. Gamma Knife radiosurgery for glomus jugulare tumors: a single-center series of 75 cases. J Neurosurg 2017;126(05): $1488-1497$

26 Marchetti M, Pinzi V, Tramacere I, Bianchi LC, Ghielmetti F, Fariselli L. Radiosurgery for paragangliomas of the head and neck: another step for the validation of a treatment paradigm. World Neurosurg 2017;98:281-287

27 Winford TW, Dorton LH, Browne JD, Chan MD, Tatter SB, Oliver ER. Stereotactic radiosurgical treatment of glomus jugulare tumors. Otol Neurotol 2017;38(04):555-562

28 Guha A, Musil Z, Vicha A, et al. A systematic review on the genetic analysis of paragangliomas: primarily focused on head and neck paragangliomas. Neoplasma 2019;66(05):671-680

29 Burnichon N, Rohmer V, Amar L, et al; PGL.NET network. The succinate dehydrogenase genetic testing in a large prospective series of patients with paragangliomas. J Clin Endocrinol Metab 2009;94(08):2817-2827 